\title{
Übergewicht erhöht Risiko für Atemwegserkrankungen
}

\author{
Zu viele Kilos gehen mit einer chronischen niedrigschwelligen systemischen \\ Entzündung einher und sind auch Risikofaktoren für Atemwegserkrankungen.
}

Insbesondere in industrialisierten Ländern nimmt Adipositas pandemisch zu, die Prävalenz hat sich in den letzten 20 Jahren verdoppelt. Die WHO schätzt, dass weltweit mehr als eine Milliarde Menschen übergewichtig sind (BMI $\geq 25 \mathrm{~kg} / \mathrm{m}^{2}$ ), davon rund 300 Millionen adipös $\left(\mathrm{BMI} \geq 30 \mathrm{~kg} / \mathrm{m}^{2}\right)$.

\section{Asthma bronchiale}

Adipöse Menschen haben ein höheres Risiko, Asthma bronchiale zu entwickeln. Außerdem können Übergewicht und Fettleibigkeit ein bestehendes Asthma verschlimmern. Offenbar senden Fettzellen ein Signal aus, das die Lungenfunktion beeinträchtigt. Inzidenz und Prävalenz von Adipositas und übergewichtsassoziiertem Asthma bronchiale steigen daher dramatisch, so Prof. Elisabeth Bel von der Universität Amsterdam beim Kongress der europäischen Pneumologengesellschaft (ERS) 2016.

Übergewichtsassoziiertes Asthma kann sowohl als atopisches „early onset“ Asthma als auch als nicht atopisches und nicht eosinophiles „late onset“ Asthma auftreten. Diese Form ist meist resistent gegen Kortikosteroide und bessert sich bei Gewichtsabnahme. Insbesondere bei adipösen Frauen ist die Prävalenz von Asthma hoch. Übergewichtsassoziiertes Asthma bei Frauen gilt als eigener Phänotyp.

Übergewichtsassoziiertes Asthma wird nicht durch eosinophile bzw. neutrophile Entzündung oder oxidativen Stress verursacht, sondern ist vielmehr die Konsequenz mechanischer Faktoren, die das Atemwegssystem belasten, etwa das Atmen mit verringertem Lungenvolumen und die mechanische Einengung der peripheren Atemwege. Dabei spielt möglicherweise auch eine reduzierte Verfügbarkeit von Stickstoffmonoxid (NO) eine Rolle. Tatsächlich zeigten Mausmodelle, dass abnorm hohes Gewicht zu einer Verengung der Bronchien und einer reduzierten Lungenfunktion führt.

Adipositas hat Konsequenzen für die Asthmatherapie, sagte Bel. Fettleibige Asthmapatienten ohne Nachweis einer eosinophilen Entzündung sollten nicht mit Kortikosteroiden behandelt werden. Dagegen sind Bronchodilatatoren (außer SABA) zu empfehlen. Therapie der Wahl ist aber eine drastische Gewichtsreduktion, auch durch eine bariatrische Operation.

\section{Obstruktive Schlafapnoe}

Übergewichtige Menschen leiden häufig an obstruktiver Schlafapnoe (OSA) mit Atemaussetzern im Schlaf. Die Erkrankung geht mit Tagesschläfrigkeit, verminderter Leistungsfähigkeit und erhöhter Unfallgefahr einher. Bei schwerer Schlafapnoe steigt die Gefahr von Folgeerkrankungen wie Bluthochdruck, Herz-Kreislauf-Störungen oder Typ-2-Diabetes.
Über die Bedeutung der CPAP-Beatmung bei OSA sprach Prof. Fernando Barbé Illa von der Universität Lleida in Katalonien/ Spanien. CPAP kann zwar kosteneffektiv die Symptome von OSA lindern und die Wachheit am Tage, die Stimmung und die Lebensqualität der $\mathrm{Be}$ troffenen verbessern, ist aber als Sekundär-

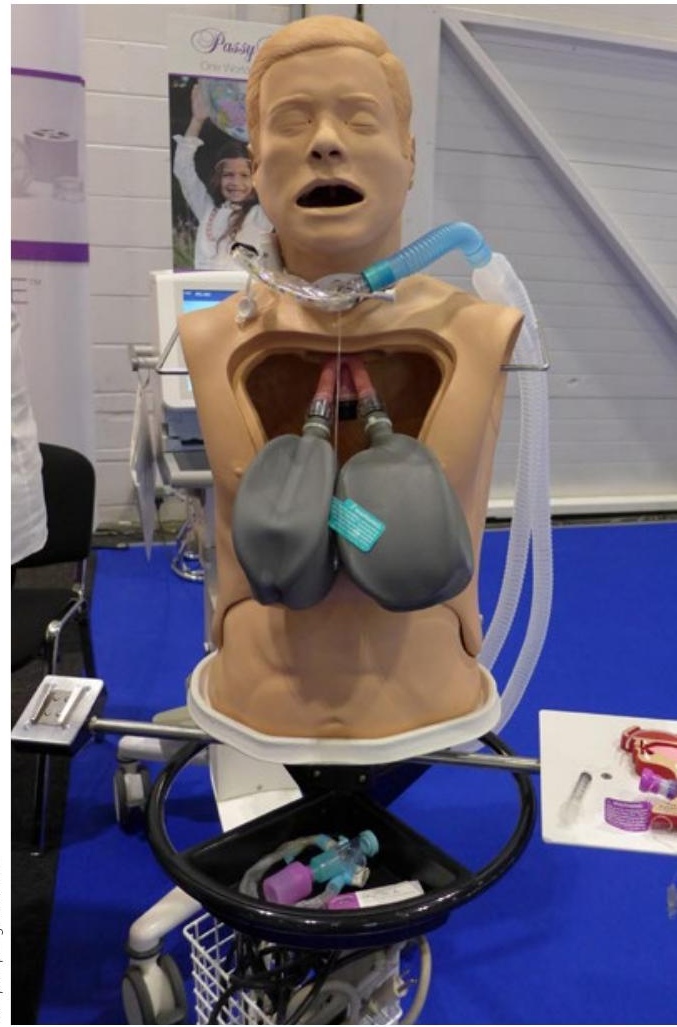

Apnoe? Puppen wie diese beim ERS-Kongress erlauben die Demonstration von Beatmungsdevices. prävention kardiovaskulärer Ereignisse bei Patienten mit koronaren oder zerebrovaskulären Erkrankungen nicht effektiv.

\section{Chronisch obstruktive Lungenerkrankung}

Ein hoher BMI erhöht nicht nur das generelle Mortalitätsrisiko, sondern ist auch speziell für eine erhöhte Sterblichkeit bei COPD mit verantwortlich, so Dr. Miguel Divo, Brigham and Women's Hospital Boston/USA. Übergewicht liegt mit hoher Prävalenz bei COPD-Patienten vor und erhöht das Risiko für einen negativen Outcome.

Während man früher dachte, dass allein ein niedriger BMI mit einer erhöhten Sterblichkeit assoziiert ist (etwa bei der eher untergewichtigen Gruppe mit ausgeprägtem Lungenemphysem („Pink Puffer“), gilt Übergewicht heute bei der übergewichtige Untergruppe („Blue Bloater“) als wichtiger Teil des Komorbidioms. Wie die Arbeitsgruppe um Divo in einer Untersuchung festgestellt hat, ist der BMI-Wert ein Krankheitsmodulator der COPD, der einen Einfluss auf den Phänotyp, die Mechanismen des Atemwegssystems sowie auf die systemische Inflammation und ihre Biomarker hat.

Die Arbeitsgruppe kommt zu dem Schluss, dass wenige Komorbiditäten signifikant mit dem Sterblichkeitsrisiko bei untergewichtigen COPD-Patienten assoziiert sind, dagegen aber viele bei hohem BMI. Komorbiditäten wie kardiovaskuläre Erkrankungen, Muskelatrophie oder metabolisches Syndrom spielen eine maßgebliche Rolle für die Sterblichkeit von COPD-Patienten, die nur in vergleichsweise wenigen Fällen an pulmonaler Insuffizienz sterben.

Dagmar Jäger-Becker

Quelle: Hot topics: Obesity and respiratory diseases, International Congress 2016 der European Respiratory Society ERS, London, 3.-7. September 2016 